\title{
Nonuniform autonomous one-dimensional exclusion nearest-neighbor reaction-diffusion models
}

\author{
Amir Aghamohammad1 1 \& Mohammad Khorrami 2 \\ Department of Physics, Alzahra University, Tehran 19384, IRAN
}

PACS numbers: 64.60.-i, 05.40.-a, 02.50.Ga

Keywords: reaction-diffusion, exclusion processes, phase transition, nonuniform reaction rates

\begin{abstract}
The most general nonuniform reaction-diffusion models on a one-dimensional lattice with boundaries, for which the time evolution equations of correlation functions are closed, are considered. A transfer matrix method is used to find the static solution. It is seen that this transfer matrix can be obtained in a closed form, if the reaction rates satisfy certain conditions. We call such models superautonomous. Possible static phase transitions of such models are investigated. At the end, as an example of superautonomous models, a nonuniform voter model is introduced, and solved explicitly.
\end{abstract}

\footnotetext{
${ }^{1}$ mohamadi@alzahra.ac.ir

${ }^{2}$ mamwad@mailaps.org
} 


\section{Introduction}

Most of the investigations on reaction-diffusion models are devoted to uniform models, where interaction rates are site-independent. Among the simplest generalizations beyond a completely uniform system is a lattice with alternating rates. In [1, relaxation in the kinetic Ising model on an alternating isotopic chain has been discussed. In 2-4, the steady state configurational probabilities of an Ising spin chain driven out of equilibrium by a coupling to two heat baths has been investigated. An example is a one-dimensional Ising model on a ring, in which the evolution is according to a generalization of Glauber rates, such that spins at even (odd) lattice sites experience a temperature $T_{\mathrm{e}}\left(T_{\mathrm{o}}\right)$. In this model the detailed balance is violated. The response function to an infinitesimal magnetic field for the Ising-Glauber model with arbitrary exchange couplings has been studied in [5]. Other generalizations of the Glauber model consist of, for example, alternating-isotopic chains and alternating-bound chains ( [6] for example). In a recent article [7, we studied the expectation values of spins in an Ising model with nonuniform coupling constants. A transfer matrix method was used to study the steady state behavior of the system in the thermodynamic limit. Different (static) phases of this system were studied, and a closed form was obtained for this transfer matrix.

In [8] a ten-parameter family of one-species reaction-diffusion processes with nearest-neighbor interaction was introduced, for which the evolution equation of $n$-point functions contains only $n$ - or less- point functions, the so called autonomous models. The average particle-number in each site was obtained exactly for these models. In [9, 10, this was generalized to multi-species systems and more-than-two-site interactions. In [11-13, the phase structure of some classes of single or multiple-species reaction-diffusion systems was investigated. These investigations were based on the one-point functions of the systems.

In the present paper the most general nonuniform exclusion nearest-neighbor reaction-diffusion models on a one-dimensional lattice with boundaries are studied, for which the evolution equations of the one-point functions are closed, and the transfer matrix has a closed form. It is shown that there is a possible phase transition in such models, which corresponds to a reduction of the role of boundary conditions on time-independent profile of the expectation value of the number operators. The scheme of the paper is as follows. In section 2, the models are introduced, and the evolution equation for the expectation values of $n_{i}$ (the number operators at the site $i$ ) is obtained. Also conditions are obtained so that the evolution of the expectation values of $n_{i}$ is closed. In section 3 , the equation governing the static solution for the expectation values of $n_{i}$ 's is obtained, and a transfer matrix method is introduced to obtain the static solution and investigate different (static) phases of the system. It is also seen that to write a closed form for the transfer matrix, further conditions on the reaction rates are to be satisfied. We call models satisfying these conditions superautonomous models. In section 4 , as an example, a nonuniform voter model is investigated in more detail. Section 5 is devoted to the concluding remarks. 


\section{Exclusion nearest-neighbor reaction-diffusion models with nonuniform reaction rates}

Consider a one-dimensional lattice with $(L+1)$ sites, numbered from 0 to $L$. Each site is either empty (denoted by the vector $e_{0}$ ) or occupied with one particle (denoted by the vector $e-1$ ). The evolution of the system is said to be governed by nearest-neighbor interactions, if the evolution of each site depends on only that site and its nearest neighbors (sites directly related to it through a link). The evolution of such a system is governed by a Hamiltonian $\mathcal{H}$ of the form,

$$
\mathcal{H}=\mathcal{H}_{0}^{\prime}+\left(\sum_{\alpha} \mathcal{H}_{\alpha}\right)+\mathcal{H}_{L}^{\prime},
$$

where $\mathcal{H}_{\alpha}$ corresponds to the link $\alpha$ :

$$
\mathcal{H}_{\alpha}=1^{\otimes(\alpha-\mu)} \otimes H_{\alpha} \otimes 1^{\otimes(L-\alpha-\mu)},
$$

and

$$
\mu:=\frac{1}{2}
$$

The link $\alpha$ links the sites $(\alpha-\mu)$ and $(\alpha+\mu)$, so that $\alpha \pm \mu$ are integers, and $\alpha$ runs from $\mu$ up to $(L-\mu)$. Throughout this paper, sites are denoted by Latin letters which represent integers, while links are denoted by Greek letters which represent integers plus one half $(\mu)$, so that the link $\alpha$ joins the sites $(\alpha-\mu)$ and $(\alpha+\mu)$, while the site $i$ joins the links $(i-\mu)$ and $(i+\mu) . H_{\alpha}$ is a linear operator acting on a four dimensional space (the configuration space corresponding to the sites $(\alpha-\mu)$ and $(\alpha+\mu))$ with a basis $\left\{e_{00}, e_{01}, e_{10}, e_{11}\right\}$. Also,

$$
\begin{aligned}
\mathcal{H}_{0}^{\prime} & =H_{0}^{\prime} \otimes 1^{\otimes L}, \\
\mathcal{H}_{L}^{\prime} & =1^{\otimes L} \otimes H_{L}^{\prime},
\end{aligned}
$$

where $H^{\prime} 0$ and $H_{L}^{\prime}$ are linear operators acting on two dimensional spaces (the configuration spaces corresponding to the sites 0 and $L$, respectively) with bases $\left\{e_{0}, e_{1}\right\}$. The nondiagonal components of $H_{\alpha}, H_{0}^{\prime}$, and $H_{L}^{\prime}$ are reaction rates. Denoting a full site by $\bullet$ and an empty site by $\circ$, the possible reactions for the boundary sites 0 or $L$ are

$$
\begin{array}{ll}
\circ \rightarrow \bullet, & \text { with the rate }\left(H_{0, L}^{\prime}\right)^{1}{ }_{0}, \\
\bullet \rightarrow \circ, & \text { with the rate }\left(H_{0, L}^{\prime}\right)^{0}{ }_{1},
\end{array}
$$


while those for the link $\alpha$ are

$$
\begin{aligned}
& \text { ○ } \rightarrow \text { ๑, with the rate }\left(H_{\alpha}\right)^{01}{ }_{00}, \\
& \text { ० } \rightarrow \bullet \circ, \quad \text { with the rate }\left(H_{\alpha}\right)^{10}{ }_{00} \text {, } \\
& \circ \circ \rightarrow \bullet \text {, with the rate }\left(H_{\alpha}\right)^{11}{ }_{00} \text {, } \\
& \circ \rightarrow \infty \text {, with the rate }\left(H_{\alpha}\right)^{00}{ }_{01} \text {, } \\
& \circ \bullet \bullet \bullet, \quad \text { with the rate }\left(H_{\alpha}\right)^{10}{ }_{01} \text {, } \\
& \bullet \bullet \bullet \bullet, \quad \text { with the rate }\left(H_{\alpha}\right)^{11}{ }_{01} \text {, } \\
& \bullet \rightarrow \infty \text {, with the rate }\left(H_{\alpha}\right)^{00}{ }_{10} \text {, } \\
& \bullet \circ \rightarrow \circ \bullet \text { with the rate }\left(H_{\alpha}\right)^{01}{ }_{10} \text {, } \\
& \bullet \rightarrow \bullet \bullet \text {, with the rate }\left(H_{\alpha}\right)^{11}{ }_{10} \text {, } \\
& \bullet \rightarrow \infty \text {, with the rate }\left(H_{\alpha}\right)^{00}{ }_{11} \text {, } \\
& \bullet \rightarrow \infty \bullet \text { with the rate }\left(H_{\alpha}\right)^{01}{ }_{11} \text {, } \\
& \bullet \bullet \bullet \bullet, \quad \text { with the rate }\left(H_{\alpha}\right)^{10}{ }_{11} \text {. }
\end{aligned}
$$

It is seen that these rates are in general different for different links (also the rates on the boundary sites are in general different). The system is called uniform if the rates are the same for all links, and nonuniform if it is not the case.

The number operator in the site $i$ is denoted by $n_{i}$ :

$$
n_{i}=1^{\otimes i} \otimes n \otimes 1^{\otimes(L-i)},
$$

where $n$ is an operator acting on a two dimensional space with the basis $\left\{e_{0}, e_{1}\right\}$. The matrix form of $n$ in this basis is

$$
n^{a}{ }_{b}=\delta_{1}^{a} \delta_{b}^{1} .
$$

The evolution equation for the expectation value of an observable $Q$ is

$$
\frac{\mathrm{d}}{\mathrm{d} t}\langle Q\rangle=\langle Q \mathcal{H}\rangle
$$

where

$$
\langle Q\rangle=S Q \Psi
$$

the vector $\Psi$ is the $\left(2^{L+1}\right.$ dimensional $)$ probability vector describing the system and $S$ is the covector

$$
S:=s^{\otimes(L+1)},
$$

and

$$
s_{a}=1 .
$$

The system is called autonomous, if the Hamiltonian is so that the evolution of the expectation values of $n_{i}$ is closed in terms of the expectation values of $n_{j}$ 's. In the evolution equation for the expectation value of $n_{i}$, the expectation values 
of $n_{i-1}, n_{i}, n_{i+1},\left(n_{i-1} n_{i}\right)$, and $\left(n_{i} n_{i+1}\right)$ occur. It is seen that the criterion that the coefficients of the last two vanish, is

$$
\begin{aligned}
& s_{a}\left[H_{i-\mu} r \otimes r\right]^{a 1}=0, \\
& s_{a}\left[H_{i+\mu} r \otimes r\right]^{1 a}=0,
\end{aligned}
$$

respectively, where

$$
r^{a}=-\delta_{0}^{a}+\delta_{1}^{a} .
$$

Equation (13) should hold for all $i$ 's, in order that the system be autonomous. So one can rewrite it like

$$
\begin{aligned}
& s_{a}\left[H_{\alpha} r \otimes r\right]^{a 1}=0, \\
& s_{a}\left[H_{\alpha} r \otimes r\right]^{1 a}=0 .
\end{aligned}
$$

It is seen that this condition is the same as the corresponding condition for uniform lattices [8, 12], written for each link separately. Provided that this condition holds, one arrives at

$\frac{\mathrm{d}}{\mathrm{d} t}\left\langle n_{i}\right\rangle=\eta_{i-\mu}\left\langle n_{i-1}\right\rangle+\theta_{i+\mu}\left\langle n_{i+1}\right\rangle+\left(\kappa_{i-\mu}+\nu_{i+\mu}\right)\left\langle n_{i}\right\rangle+\left(\xi_{i-\mu}+\sigma_{i+\mu}\right), \quad 0<i<L$,

where

$$
\begin{aligned}
\eta_{\alpha} & :=s_{a}\left(H_{\alpha}\right)^{a 1}{ }_{b 0} r^{b}, \\
\theta_{\alpha} & :=s_{a}\left(H_{\alpha}\right)^{1 a_{0 b}} r^{b}, \\
\kappa_{\alpha} & :=s_{a}\left(H_{\alpha}\right)^{a 1}{ }_{0 b} r^{b}, \\
\nu_{\alpha} & :=s_{a}\left(H_{\alpha}\right)^{1 a_{b 0}} r^{b}, \\
\xi_{\alpha} & :=s_{a}\left(H_{\alpha}\right)^{a 1}{ }_{00}, \\
\sigma_{\alpha} & :=s_{a}\left(H_{\alpha}\right)^{1 a_{00} .}
\end{aligned}
$$

For the boundary sites (the sites 0 and $L$ ), one has

$$
\begin{aligned}
& \frac{\mathrm{d}}{\mathrm{d} t}\left\langle n_{0}\right\rangle=\theta_{\mu}\left\langle n_{1}\right\rangle+\left[\left(H_{0}^{\prime}\right)^{1}{ }_{1}-\left(H_{0}^{\prime}\right)^{0}{ }_{1}+\nu_{\mu}\right]\left\langle n_{0}\right\rangle+\left[\left(H_{0}^{\prime}\right)^{0}{ }_{1}+\sigma_{\mu}\right] \\
& \frac{\mathrm{d}}{\mathrm{d} t}\left\langle n_{L}\right\rangle=\eta_{L-\mu}\left\langle n_{L-1}\right\rangle+\left[\kappa_{L-\mu}+\left(H_{L}^{\prime}\right)^{1}{ }_{1}-\left(H_{L}^{\prime}\right)^{0}{ }_{1}\right]\left\langle n_{L}\right\rangle+\left[\xi_{L-\mu}+\left(H_{L}^{\prime}\right)^{0}{ }_{1}\right]
\end{aligned}
$$

\section{The static solution}

For the static solution $\left(\langle n\rangle_{\mathrm{st}}\right)$, the left hand side of (16) vanishes and one obtains

$$
\left\langle n_{i+1}\right\rangle_{\mathrm{st}}=-\frac{\eta_{i-\mu}}{\theta_{i+\mu}}\left\langle n_{i-1}\right\rangle_{\mathrm{st}}-\frac{\kappa_{i-\mu}+\nu_{i+\mu}}{\theta_{i+\mu}}\left\langle n_{i}\right\rangle_{\mathrm{st}}-\frac{\xi_{i-\mu}+\sigma_{i+\mu}}{\theta_{i+\mu}} .
$$


Denoting that part of this solution which satisfies the homogeneous equation by $\langle n\rangle_{\mathrm{st}}^{\mathrm{hom}}$, it is seen that

$$
\left\langle n_{i+1}\right\rangle_{\mathrm{st}}^{\mathrm{hom}}=-\frac{\eta_{i-\mu}}{\theta_{i+\mu}}\left\langle n_{i-1}\right\rangle_{\mathrm{st}}^{\mathrm{hom}}-\frac{\kappa_{i-\mu}+\nu_{i+\mu}}{\theta_{i+\mu}}\left\langle n_{i}\right\rangle_{\mathrm{st}}^{\mathrm{hom}},
$$

which can be written as the following matrix form

$$
X_{i+\mu}^{\mathrm{hom}}=D_{i} X_{i-\mu}^{\mathrm{hom}}
$$

where

$$
X_{\alpha}:=\left[\begin{array}{l}
\left\langle n_{\alpha-\mu}\right\rangle_{\mathrm{st}} \\
\left\langle n_{\alpha+\mu}\right\rangle_{\mathrm{st}}
\end{array}\right]
$$

and

$$
D_{i}:=\left[\begin{array}{cc}
0 & 1 \\
-\frac{\eta_{i-\mu}}{\theta_{i+\mu}} & -\frac{\kappa_{i-\mu}+\nu_{i+\mu}}{\theta_{i+\mu}}
\end{array}\right] .
$$

Using these, one arrives at

$$
X_{\alpha}^{\mathrm{hom}}=D_{\alpha \beta} X_{\beta}^{\mathrm{hom}}
$$

where

$$
D_{\alpha \beta}:=D_{\alpha-\mu} D_{\alpha-\mu-1} \cdots D_{\beta+\mu} .
$$

To solve (20), one can use a tranfer matrix (Green's function) method. Consider the equation

$$
G_{i+1 j}=-\frac{\eta_{i-\mu}}{\theta_{i+\mu}} G_{i-1 j}-\frac{\kappa_{i-\mu}+\nu_{i+\mu}}{\theta_{i+\mu}} G_{i j}-\delta_{i j} .
$$

Defining

$$
Y_{\alpha j}:=\left[\begin{array}{l}
G_{\alpha-\mu j} \\
G_{\alpha+\mu j}
\end{array}\right]
$$

it is seen that the solution for $Y$ is

$$
Y_{\alpha j}=\left\{\begin{array}{ll}
D_{\alpha \beta} \tilde{Y}_{\beta j}, & \alpha<j \\
D_{\alpha \beta} Y_{\beta j}, & \alpha>j
\end{array},\right.
$$

with the condition that (27) holds for $i=j$. This condition is

$$
Y_{j+\mu j}=D_{j} Y_{j-\mu j}-\left[\begin{array}{l}
0 \\
1
\end{array}\right],
$$

which reads

$$
D_{j+\mu \beta}\left(Y_{\beta j}-\tilde{Y}_{\beta j}\right)=-\left[\begin{array}{l}
0 \\
1
\end{array}\right] .
$$


A particular solution for $Y$ is obtained if one sets $\tilde{Y}$ equal to zero. In this case,

$$
Y_{\alpha j}=-\Theta_{\alpha j} D_{\alpha j+\mu}\left[\begin{array}{l}
0 \\
1
\end{array}\right],
$$

where $\Theta$ is the step function:

$$
\Theta_{\alpha j}:=\left\{\begin{array}{ll}
0, & \alpha<j \\
1, & \alpha>j
\end{array} .\right.
$$

Using this, the general solution to (20) can be written as

$$
X_{\alpha}=D_{\alpha \beta} X_{\beta}^{\mathrm{hom}}-\sum_{j<\alpha} D_{\alpha j+\mu} \frac{\xi_{j-\mu}+\sigma_{j+\mu}}{\theta_{j+\mu}}\left[\begin{array}{l}
0 \\
1
\end{array}\right] .
$$

As it was the case in [7, the steady state profile near the end-site 0 is determined by the eigenvalues of the matrix $D_{\alpha \mu}$, where $\alpha$ is some site far from the ends. One has

$$
\begin{aligned}
X_{\alpha} & =X_{\alpha}^{a} \mathbf{f}_{a}, \\
X_{\mu} & =X_{\mu}^{a} \mathbf{f}_{a},
\end{aligned}
$$

where $\mathbf{f}_{a}$ is the eigenvector of $D_{\alpha \mu}$ corresponding to the eigenvalue $\lambda^{a}$, and $X_{\alpha}^{a}$ 's and $X_{\mu}^{a}$ 's are the coefficients of expansions of $X_{\alpha}$ and $X_{\mu}$ in terms of the eigenvectors. If there was no nonhomogeneous part in the equation (16), then the discussion would be exactly similar to that of [7]: $X_{\mu}^{a}$ vanishes if $\lambda^{a}$ tends to infinity (in the thermodynamic limit). Otherwise, $X_{\mu}^{a}$ is generally nonzero and determined by the boundary conditions. If the nonhomogeneous part does not vanish, then the second term in (34) generally contains a large multiple of $\mathbf{f}_{a}$ if $\lambda^{a}$ tends to infinity. This large part is to be cancelled by a large multiple of $\mathbf{f}_{a}$ coming from the first term in (34). So in this case $X_{\mu}^{a}$ does not vanish but tends to a fixed value independent of boundary conditions. It is seen that although equations (18) and (19) serve as boundary conditions to obtain say $X_{\mu}$, the above general argument is independent of these conditions. The essence of the above argument is the following. In general, $X_{\alpha}$ (the components of which are noting but expectations of number operators) is a linear combination of two vectors $\left(\mathbf{f}_{a}\right.$ 's) plus a nonhomogeneous part. The nonhomogeneous part is determined from the bulk reactions, and the two unknown coefficients $\mathbf{f}_{a}$ 's are to be determined from the the boundary conditions resulted from (18) and (19) (in the static case that the left hand sides vanish). There are, however, regions of the parameter space where in the thermodynamic limit one of the coefficients of $\mathbf{f}_{a}$ 's (or possibly both) are determined from the bulk reactions only. This essentially means that the effect of boundaries on the behavior of the system is reduced. In a transport system, for example, it is expected that the time-independent profile of the moving bodies' density depend on both the bulk reactions (speed, overtaking, etc) and the boundary reactions (injection and extraction rates). But there could be cases where these boundary terms are unimportant, or less important.

The situation (in the thermodynamic limit) can be summarized as follows. 
i The eigenvalue $\lambda^{a}$ tends to infinity. In this case $X_{\mu}^{a}$ tends to a fixed value, independent of the boundary conditions.

ii The eigenvalue $\lambda^{a}$ tends to zero or a finite number. In this case $X_{\mu}^{a}$ is determined by the boundary condiotions.

Obviously, similar cases occur at the other boundary site. It is seen that this behavior at one of the boundaries is independent of the analog behavior at the other boundary.

This is the static phase transition of the system, some discontinuous behavior of the expectation value of the number operator near the boundaries (note that the components of $X_{\alpha}$ are nothing but the expectations of number operators).

If one can write $D_{i}$ as

$$
D_{i}:=\Sigma_{i+\mu} \Delta_{i} \Sigma_{i-\mu}^{-1},
$$

where $\Delta_{i}$ is diagonal, and $\Sigma_{\alpha}$ depends on only the parameters corresponding to the link $\alpha$, then it is easy to find the solution to (22). The system is called superautonomous, if this is the case. Putting

$$
\Sigma_{\alpha}=\left[\begin{array}{ll}
a_{\alpha} & b_{\alpha} \\
c_{\alpha} & d_{\alpha}
\end{array}\right]
$$

and

$$
\Delta_{i}=\left[\begin{array}{cc}
A_{i} & 0 \\
0 & B_{i}
\end{array}\right],
$$

in (36), one arrives at

$$
\begin{aligned}
\frac{A_{i}}{\varsigma_{i-\mu}} a_{i+\mu} d_{i-\mu}-\frac{B_{i}}{\varsigma_{i-\mu}} b_{i+\mu} c_{i-\mu} & =0, \\
-\frac{A_{i}}{\varsigma_{i-\mu}} a_{i+\mu} b_{i-\mu}+\frac{B_{i}}{\varsigma_{i-\mu}} b_{i+\mu} a_{i-\mu} & =1, \\
\frac{A_{i}}{\varsigma_{i-\mu}} c_{i+\mu} d_{i-\mu}-\frac{B_{i}}{\varsigma_{i-\mu}} d_{i+\mu} c_{i-\mu} & =-\frac{\eta_{i-\mu}}{\theta_{i+\mu}}, \\
-\frac{A_{i}}{\varsigma_{i-\mu}} c_{i+\mu} b_{i-\mu}+\frac{B_{i}}{\varsigma_{i-\mu}} d_{i+\mu} a_{i-\mu} & =-\frac{\kappa_{i-\mu}+\nu_{i+\mu}}{\theta_{i+\mu}},
\end{aligned}
$$

where

$$
\varsigma_{\alpha}:=a_{\alpha} d_{\alpha}-b_{\alpha} c_{\alpha}
$$

Using (39) and (40), one obtains $A_{i}$ and $B_{i}$ :

$$
\begin{aligned}
A_{i} & =\frac{c_{i-\mu}}{a_{i+\mu}}, \\
B_{i} & =\frac{d_{i-\mu}}{b_{i+\mu}} .
\end{aligned}
$$


Using these in (41), it is seen that

$$
\frac{c_{i-\mu} d_{i-\mu}}{\varsigma_{i-\mu}} \frac{\varsigma_{i+\mu}}{a_{i+\mu} d_{i+\mu}}=\frac{\eta_{i-\mu}}{\theta_{i+\mu}}
$$

which can be solved as

$$
\begin{aligned}
& a_{\alpha} b_{\alpha}=\phi \theta_{\alpha} \varsigma_{\alpha}, \\
& c_{\alpha} d_{\alpha}=\phi \eta_{\alpha} \varsigma_{\alpha},
\end{aligned}
$$

where $\phi$ is a constant. Finally, using (44), (45), (47), and (48) in (42), on obtains

$$
\frac{a_{i-\mu} d_{i-\mu}}{\varsigma_{i-\mu}}+\frac{b_{i+\mu} c_{i+\mu}}{\varsigma_{i+\mu}}=-\phi \kappa_{i-\mu}-\phi \nu_{i+\mu},
$$

the solution to which is

$$
\begin{aligned}
a_{\alpha} d_{\alpha} & =\left(-\phi \kappa_{\alpha}+\psi\right) \varsigma_{\alpha}, \\
b_{\alpha} c_{\alpha} & =\left(-\phi \nu_{\alpha}-\psi\right) \varsigma_{\alpha},
\end{aligned}
$$

where $\psi$ is another constant. $\varsigma$ is not an independent variable in $\Sigma$. Putting (50) and (51) in (43), one arrives at

$$
2 \psi=\phi\left(\kappa_{\alpha}-\nu_{\alpha}\right)+1
$$

which shows that $(\kappa-\nu)$ should be constant. One can use (52) in (50) and (51), to obtain

$$
\begin{aligned}
a_{\alpha} d_{\alpha} & =\frac{\varsigma_{\alpha}}{2}\left[-\phi\left(\kappa_{\alpha}+\nu_{\alpha}\right)+1\right], \\
b_{\alpha} c_{\alpha} & =\frac{\varsigma_{\alpha}}{2}\left[-\phi\left(\kappa_{\alpha}+\nu_{\alpha}\right)-1\right] .
\end{aligned}
$$

These two equations are not independent of (47) and (48). The consistency condition is

$$
\phi^{2}\left(\kappa_{\alpha}+\nu_{\alpha}\right)^{2}-1=4 \phi^{2} \eta_{\alpha} \theta_{\alpha}
$$

showing that $\left[(\kappa+\nu)^{2}-4 \eta \theta\right]$ should be constant. Noting that $(\kappa-\nu)$ has to be a constant as well, the second condition can be stated as $(\kappa \nu-\eta \theta)$ should be constant. So the conditions that the system be superautonomous are

$$
\begin{aligned}
\kappa_{\alpha}-\nu_{\alpha} & =\text { constant } \\
\kappa_{\alpha} \nu_{\alpha}-\eta_{\alpha} \theta_{\alpha} & =\text { constant. }
\end{aligned}
$$

There are some special cases resembling those encountered in [7].

1 Constant coupling: Here $H_{\alpha}$ does not depend on $\alpha$, and $\lambda^{a}$ tends to infinity (zero) if and only if the corresponding eigenvalue of $D_{i}$ is greater (smaller) than one. 
2 Periodic coupling:

$$
H_{\alpha+m}=H_{\alpha} .
$$

In this case the behavior of the eigenvalues of $D_{\alpha, \mu}$ is determined by the eigenvalues of $D_{\alpha+m, \alpha}$ : An eigenvalue of $D_{\alpha, \mu}$ tends to infinity (zero) if and only if the corresponding eigenvalue of $D_{\alpha+m, \alpha}$ is greater (smaller) than one.

3 Defects in the lattice: No new phenomena is seen, as long as the defects are localized, i.e. they are far from the boundaries. So if there is a lattice that has some defects but otherwise is uniform, the static behavior near the boundaries is similar to that of a uniform lattice [12].

4 A lattice with different behaviors at different end points: The behaviors of the static solution near the two ends are independent of each other, provided the behavior change occurs far from the boundaries. So all the phenomena seen in previous special cases can be seen at each boundary, independent of the other boundary.

\section{An example, the voter model}

The voter model is a lattice each site of which is either full $(\bullet)$ or empty (०). The reactions on a link are

$$
\begin{array}{ll}
\bullet \rightarrow \infty, & \text { with the rate } u, \\
\bullet \rightarrow \bullet \bullet, & \text { with the rate } v, \\
\bullet \bullet \rightarrow \bullet \bullet & \text { with the rate } u, \\
\bullet \bullet \rightarrow \infty, & \text { with the rate } v .
\end{array}
$$

Of course, the rates $u$ and $v$ may be link-dependent. using (17),

$$
\begin{aligned}
\eta_{\alpha} & =v_{\alpha}, \\
\theta_{\alpha} & =u_{\alpha}, \\
\kappa_{\alpha} & =-v_{\alpha}, \\
\nu_{\alpha} & =-u_{\alpha}, \\
\xi_{\alpha} & =0, \\
\sigma_{\alpha} & =0 .
\end{aligned}
$$

In order that the system be superautonomous, (56) and (57) must hold. (56) reads

$$
u_{\alpha}-v_{\alpha}=\text { constant },
$$

and (57) is an identity. Assume that (61) holds. Using (24), one has

$$
D_{i}:=\left[\begin{array}{cc}
0 & 1 \\
-\frac{v_{i-\mu}}{u_{i+\mu}} & 1+\frac{v_{i-\mu}}{u_{i+\mu}}
\end{array}\right] .
$$


From (55), one has

$$
\phi^{2}\left(u_{\alpha}-v_{\alpha}\right)^{2}=1,
$$

one of the solutions to which is

$$
\phi=\frac{1}{u_{\alpha}-v_{\alpha}} .
$$

Putting this in (47), (48), (53), and (54), one arrives at

$$
\begin{gathered}
a_{\alpha} b_{\alpha}=\phi u_{\alpha} \varsigma_{\alpha}, \\
c_{\alpha} d_{\alpha}=\phi v_{\alpha} \varsigma_{\alpha}, \\
a_{\alpha} d_{\alpha}=\phi u_{\alpha} \varsigma_{\alpha}, \\
b_{\alpha} c_{\alpha}=\phi v_{\alpha} \varsigma_{\alpha} .
\end{gathered}
$$

One set of solutions to these equations is

$$
\Sigma_{\alpha}=\left[\begin{array}{ll}
u_{\alpha} & 1 \\
v_{\alpha} & 1
\end{array}\right] .
$$

Putting this in (44) and (45) results in

$$
\Delta_{i}=\left[\begin{array}{cc}
\frac{v_{i-\mu}}{u_{i+\mu}} & 0 \\
0 & 1
\end{array}\right]
$$

One also has

$$
\begin{aligned}
\varsigma_{\alpha} & =u_{\alpha}-v_{\alpha}, \\
& =\phi^{-1} .
\end{aligned}
$$

So

$$
D_{\alpha \mu}=\left[\begin{array}{cc}
\phi v_{\mu}\left(\frac{v_{\alpha-1} \cdots v_{\mu+1}}{u_{\alpha-1} \cdots u_{\mu+1}}-1\right) & 1-\phi v_{\mu}\left(\frac{v_{\alpha-1} \cdots v_{\mu+1}}{u_{\alpha-1} \cdots u_{\mu+1}}-1\right) \\
\phi v_{\mu}\left(\frac{v_{\alpha} \cdots v_{\mu+1}}{u_{\alpha} \cdots u_{\mu+1}}-1\right) & 1-\phi v_{\mu}\left(\frac{v_{\alpha} \cdots v_{\mu+1}}{u_{\alpha} \cdots u_{\mu+1}}-1\right)
\end{array}\right] .
$$

One has

$$
\begin{aligned}
\operatorname{det}\left(D_{\alpha \mu}\right) & =\frac{v_{\mu}}{u_{\alpha}}\left(\frac{v_{\alpha-1} \cdots v_{\mu+1}}{u_{\alpha-1} \cdots u_{\mu+1}}\right), \\
\operatorname{tr}\left(D_{\alpha \mu}\right) & =1+\frac{v_{\mu}}{u_{\alpha}}\left(\frac{v_{\alpha-1} \cdots v_{\mu+1}}{u_{\alpha-1} \cdots u_{\mu+1}}\right),
\end{aligned}
$$

showing that the eigenvalues of $D_{\alpha \mu}$ are

$$
\begin{aligned}
& \lambda^{1}=1, \\
& \lambda^{2}=\frac{v_{\mu}}{u_{\alpha}}\left(\frac{v_{\alpha-1} \cdots v_{\mu+1}}{u_{\alpha-1} \cdots u_{\mu+1}}\right) .
\end{aligned}
$$


A special case is when

$$
v_{\alpha}=u_{\alpha} .
$$

In this case the matrix $\Sigma_{\alpha}$ is singular. Yet one can obtain the matrix $D_{\alpha \mu}$ as a limit $\phi \rightarrow \infty$ of (72). The result is

$$
D_{\alpha \mu}=\left[\begin{array}{cc}
-v_{\mu}\left(\frac{1}{v_{\alpha-1}}+\cdots+\frac{1}{v_{\mu+1}}\right) & 1+v_{\mu}\left(\frac{1}{v_{\alpha-1}}+\cdots+\frac{1}{v_{\mu+1}}\right) \\
-v_{\mu}\left(\frac{1}{v_{\alpha}}+\cdots+\frac{1}{v_{\mu+1}}\right) & 1+v_{\mu}\left(\frac{1}{v_{\alpha}}+\cdots+\frac{1}{v_{\mu+1}}\right)
\end{array}\right],
$$

and both eigenvalues of $D_{\alpha \mu}$ become one.

Let's study some special cases.

1 Constant coupling:

$$
u_{\alpha}=\text { constant }
$$

In this case $\lambda^{2}$ tends to infinity if and only if $v_{\alpha}$ is greater than $u_{\alpha}$.

2 Periodic coupling:

$$
u_{\alpha+m}=u_{\alpha} .
$$

In this case the behavior of the eigenvalues of $D_{\alpha, \mu}$ is determined by the eigenvalues of $D_{\alpha+m, \alpha}$, which are one and

$$
\Lambda^{2}=\frac{v_{\mu+m-1} \cdots v_{\mu}}{u_{\mu+m-1} \cdots u_{\mu}} .
$$

If $u_{\alpha}$ is greater than $v_{\alpha}$, then $\lambda^{2}$ tends to zero. If $u_{\alpha}$ is smaller than $v_{\alpha}$, then $\lambda^{2}$ tends to infinity.

3 closed lattice: Let the $L^{\prime}$ th site be the same as the 0'th site. One has

$$
x_{L+\mu}=x_{\mu} .
$$

Combining this with (34), it is seen that $x_{\mu}$ should be the eigenvector of $D_{L+\mu, \mu}$ corresponding to the eigenvalue one, which shows that

$$
x_{\mu}=\left[\begin{array}{l}
1 \\
1
\end{array}\right],
$$

showing that the stationary profile of the density is uniform. This is despite the fact that the reaction rates are not necessarily uniform.

\section{Concluding remarks}

General autonomous exclusion models with nearest-neighbor interactions on a one-dimensional lattice were studied, for them the reaction rates were nonuniform. By autonomous is is meant that the evolution equation for the expectation values of the number operators are closed. It was seen that the condition that 
the system be autonomous is the same as the analogous condition for uniform lattices. A transfer matrix method was introduced to solve the equation for the static configuration of the expectation values of the number operators. The static phase picture of these systems, including possible phase transitions, was investigated. These phase transitions correspond to a reduction of the role of boundary reactions on the profile of the expectations of the number operators, similar to the case of uniform lattices. Also similar to the case of uniform lattices, these possible phase transitions are not affected by the boundary conditions. Moreover, all of the above mentioned possible static phase transitions near one boundary, are controlled by only the bulk reaction rates in a large part of the lattice one boundary of which is the same boundary. So phase transitions at different boundaries are independent of each other, and finite defects far from boundaries have no effect on the phase transitions. It was seen that if the reaction rates satisfy ceratin additional conditions (which are essentially the constancy of ceratin combinations of reaction rates) a closed form can be obtained for the transfer matrix. Systems satisfying those conditions were called superautonomous. The example of the voter model was studied in more detail.

Acknowledgement: This work was partially supported by the research council of the Alzahra University. 


\section{References}

[1] L. L. Gonçalves, M. López de Haro, J. Tagüeña-Martínez, \& R. B. Stinchcombe; arXiv:cond-mat/9911225

[2] B. Schmittmann \& F. Schmüser; Phys. Rev. E66 (2002) 046130.

[3] B. Schmittmann \& F. Schmüser; J. Phys. A35 (2002) 2569.

[4] M. Mobilia, R. K. P. Zia, \& B. Schmittmann; J. Phys. A37 (2004) L407.

[5] C. Chatelain; J. Phys. A36 (2003) 10739.

[6] L. L. Gonçalves \& A. L. Stella; J. Phys. A20 (1987) L387.

[7] M. Khorrami \& A. Aghamohammadi; arXiv:0811.2283.

[8] G. M. Schütz; J. Stat. Phys. 79 (1995) 243.

[9] A. Aghamohammadi, A. H. Fatollahi, M. Khorrami, \& A. Shariati; Phys. Rev. E62 (2000) 4642.

[10] A. Shariati, A. Aghamohammadi, \& M. Khorrami; Phys. Rev. E64 (2001) 066102.

[11] M. Khorrami \& A. Aghamohammadi; Phys. Rev. E63 (2001) 042102.

[12] A. Aghamohammadi \& M. Khorrami; J. Phys. A34 (2001) 7431.

[13] M. Khorrami \& A. Aghamohammadi; Phys. Rev. E65 (2002) 056129. 